\title{
HARDY-SOBOLEV INEQUALITIES FOR SOBOLEV FUNCTIONS IN CENTRAL HERZ-MORREY SPACES ON THE UNIT BALL
}

\section{Yoshihiro Mizuta and Tetsu Shimomura}

Hiroshima University, Grad. School of Advanced Science and Engineering, Dept. of Mathematics Higashi-Hiroshima 739-8521, Japan; yomizuta@hiroshima-u.ac.jp

Hiroshima University, Grad. School of Humanities and Social Sciences, Dept. of Mathematics Higashi-Hiroshima 739-8524, Japan; tshimo@hiroshima-u.ac.jp

\begin{abstract}
Our aim in this paper is to establish Hardy-Sobolev inequalities for Sobolev functions and generalized Riesz potentials in central Herz-Morrey spaces on the unit ball. As an application, we obtain norm inequalities for Green potentials.
\end{abstract}

\section{Introduction}

The classical Hardy inequality says that if $u \in C^{1}\left(\mathbf{R}^{N}\right)$ is a function such that $u=0$ outside the unit ball $\mathbf{B}$, then

$$
\int_{\mathbf{B}}|u(x)|^{p}(1-|x|)^{-p+\beta} d x \leq\left(\frac{p}{p-1-\beta}\right)^{p} \int_{\mathbf{B}}|\nabla u(x)|^{p}(1-|x|)^{\beta} d x,
$$

where $p \geq 1$ and $\beta<p-1$. When $\beta=p-1$, (1.1) is replaced by

$$
\begin{aligned}
& \int_{\mathbf{B}}|u(x)|^{p}(1-|x|)^{-1}(\log (1 /(1-|x|)))^{-p} d x \\
& \leq\left(\frac{p}{p-1}\right)^{p} \int_{\mathbf{B}}|\nabla u(x)|^{p}(1-|x|)^{p-1}|x|^{1-N} d x
\end{aligned}
$$

for $u \in C^{1}(\mathbf{B})$ such that $u(0)=0$; see e.g. $[9,11,12,13]$. The proofs of those facts can be done by the fundamental theorem of calculus and Hölder's inequality (see the Appendix).

Let $B(x, r)=\left\{y \in \mathbf{R}^{N}:|y-x|<r\right\}, \mathbf{B}=B(0,1)$ and $A(r)=\{y \in \mathbf{B}: r \leq$ $1-|y|<2 r\}$ for $x \in \mathbf{R}^{N}$ and $r>0$. To obtain general results, as an extension of the Lebesgue $L^{p}$ space, for $p \geq 1, q>0$ and a weight $\omega$, we consider the central Herz-Morrey space

$$
H^{p, q, \omega}(\mathbf{B})=\left\{f \in L_{\mathrm{loc}}^{1}(\mathbf{B}):\|f\|_{H^{p, q, \omega}(\mathbf{B})}<\infty\right\},
$$

where

$$
\|f\|_{H^{p, q, \omega}(\mathbf{B})}=\left(\int_{0}^{1}\left(\omega(r)\|f\|_{L^{p}(A(r))}\right)^{q} \frac{d r}{r}\right)^{1 / q}
$$

in case $q<\infty$ and

$$
\|f\|_{H^{p, \infty, \omega}(\mathbf{B})}=\sup _{0<r<1} \omega(r)\|f\|_{L^{p}(A(r))}
$$

in case $q=\infty$. When $\omega(r)=r^{\beta}$, we write $H^{p, q, \beta}(\mathbf{B})$ for $H^{p, q, \omega}(\mathbf{B})$. It is well known that Morrey spaces and Herz spaces play an important role in harmonic analysis and PDE; see e.g. [6, 10, 19]. Further, see [2] for Morrey spaces, [3, 4, 21] for

https://doi.org/10.5186/aasfm.2021.4662

2020 Mathematics Subject Classification: Primary 46E30; Secondary 31B15, 26D10.

Key words: Hardy-Sobolev inequality, central Herz-Morrey spaces, Riesz potentials. 
local Morrey spaces, [7, 8] for complementary local Morrey-type spaces, [15, 16, 17] for Herz-Morrey spaces, [5] for the summability of Fourier transforms, [20] for the regularity theory for elliptic equations in divergence form and so on.

Our first aim in this paper is to extend (1.1) to the central Herz-Morrey case. In fact, we shall show that $u \in H^{p, q,-1+\beta}(\mathbf{B})$ when $p \geq 1, \beta<(p-1) / p=1 / p^{\prime}$ and $u \in C^{1}\left(\mathbf{R}^{N}\right)$ is a function such that $u=0$ outside $\mathbf{B}$ and $|\nabla u| \in H^{p, q, \beta}(\mathbf{B})$ (Theorem 2.2). We also treat the case when $\beta=1 / p^{\prime}$ (Theorem 2.3).

In Section 3, we give Hardy-Sobolev inequalities for Sobolev functions in $C_{0}^{1}(\mathbf{B})$ (Theorems 3.1 and 3.4).

Following [18], for $m \geq 0$ let us consider

$$
K_{\alpha, m}(x, y)=c(\alpha, N) \times\left\{\begin{array}{lc}
|x-y|^{\alpha-N} & \text { when } y \in B(0,1 / 2), \\
|x-y|^{\alpha-N}-\sum_{\ell=0}^{m}\left(1-|y|^{2}\right)^{\ell} \phi_{\alpha, \ell}\left(x, y^{*}\right) & \text { when } y \in \mathbf{B} \backslash B(0,1 / 2),
\end{array}\right.
$$

where $0<\alpha<N$ and $c(\alpha, N)=1 /\left((N-\alpha) \omega_{N-1}\right)$ with $\omega_{N-1}$ denoting the area of the unit sphere; for the precise definition, see Section 4 . For $f \in L_{\text {loc }}^{1}\left(\mathbf{R}^{N}\right)$, we consider the Riesz potential

$$
I_{\alpha, m} f(x)=\int_{\mathbf{B}} K_{\alpha, m}(x, y) f(y) d y
$$

whenever the integrals are well-defined.

Our second aim is to establish norm inequalities for the operators $f \rightarrow I_{\alpha, m} f$ from one central Herz-Morrey space $H^{p, q, \beta}(\mathbf{B})$ to another central Herz-Morrey space $H^{p_{\lambda}, q, \lambda-\alpha+\beta}(\mathbf{B})$ when $0 \leq \lambda \leq \alpha, 1 / p_{\lambda}=1 / p-\lambda / N$ (see Theorems 4.9 and 4.13 below). The case $\lambda=\alpha$ and $\lambda=0$ give (weighted) Sobolev's inequality and Hardy's inequality, respectively. For Riesz potentials $I_{\alpha} f(x)=\int_{\mathbf{B}}|x-y|^{\alpha-N} f(y) d y$, see Corollaries 4.11 and 4.12 .

Let $G(x, y)$ be a Green kernel on $\mathbf{B}(N \geq 3)$. We define the Green potential for $f \in L_{\mathrm{loc}}^{1}(\mathbf{B})$ by

$$
G f(x)=\int_{\mathbf{B}} G(x, y) f(y) d y .
$$

As an application, we shall show norm inequalities for Green potentials $G f$ in our settings (Corollary 4.15).

\section{Hardy's inequality}

Throughout this paper, let $C$ denote various positive constants independent of the variables in question.

If $u \in C^{1}\left(\mathbf{R}^{N}\right)$ is a function such that $u=0$ outside $\mathbf{B}$, then

$$
u(x)=-\int_{1}^{\infty} \frac{d}{d r} u(r x) d r,
$$

so that

$$
|u(x)| \leq \int_{1}^{\infty}|x||\nabla u(r x)| d r .
$$

Lemma 2.1. Let $p \geq 1$ and $\beta<1 / p^{\prime}$. Then there exists a constant $C>0$ such that

$$
\int_{\mathbf{B}}|u(x)|^{p} d x \leq C
$$


Hardy-Sobolev inequalities for Sobolev functions in central Herz-Morrey spaces on the unit ball 1033 when $u \in C^{1}\left(\mathbf{R}^{N}\right)$ is a function such that $u=0$ outside $\mathbf{B}$ and $\||\nabla u|\|_{H^{p, q, \beta}(\mathbf{B})} \leq 1$.

Proof. Let $u \in C^{1}\left(\mathbf{R}^{N}\right)$ be a function such that $u=0$ outside $\mathbf{B}$ and $\||\nabla u|\|_{H^{p, q, \beta}(\mathbf{B})}$ $\leq 1$. If $p>1$, for $x \in \mathbf{B}$, we have by (2.1) and Hölder's inequality

$$
\begin{aligned}
|u(x)| & \leq|x|\left(\int_{1}^{\infty}|\nabla u(r x)|^{p}(1-r|x|)^{a p} d r\right)^{1 / p}\left(\int_{1}^{1 /|x|}(1-r|x|)^{-a p^{\prime}} d r\right)^{1 / p^{\prime}} \\
& \leq|x|\left(\int_{1}^{\infty}|\nabla u(r x)|^{p}(1-r|x|)^{a p} d r\right)^{1 / p}\left(\frac{1}{1-a p^{\prime}} \frac{(1-|x|)^{-a p^{\prime}+1}}{|x|}\right)^{1 / p^{\prime}} \\
& \leq c_{1}|x|^{1 / p}\left(\int_{1}^{\infty}|\nabla u(r x)|^{p}(1-r|x|)^{a p} d r\right)^{1 / p}
\end{aligned}
$$

when $1-a p^{\prime}>0$, where $c_{1}=\left(1-a p^{\prime}\right)^{-1 / p^{\prime}}$. Hence we obtain by Fubini's theorem

$$
\begin{aligned}
\|u\|_{L^{p}(\mathbf{B})} & \leq C\left(\int_{1}^{\infty}\left(\int_{\mathbf{B}}|\nabla u(r x)|^{p}|x|(1-r|x|)^{a p} d x\right) d r\right)^{1 / p} \\
& \leq C\left(\int_{1}^{\infty}\left(\int_{\mathbf{B}}|\nabla u(y)|^{p}(1-|y|)^{a p}|y| r^{-1-N} d y\right) d r\right)^{1 / p} \\
& \leq C\left(\int_{\mathbf{B}}|\nabla u(y)|^{p}(1-|y|)^{a p}\left(\int_{1}^{\infty} r^{-1-N} d r\right) d y\right)^{1 / p} \\
& \leq C\left(\int_{\mathbf{B}}|\nabla u(y)|^{p}(1-|y|)^{a p} d y\right)^{1 / p} \\
& \leq C \sum_{j=0}^{\infty}\left(2^{-j}\right)^{a}\||\nabla u|\|_{L^{p}\left(A\left(2^{-j}\right)\right)} .
\end{aligned}
$$

This remains true for $p=1$.

If $1<q<\infty$, for $\beta<a<1 / p^{\prime}$, we obtain by Hölder's inequality

$$
\begin{aligned}
\|u\|_{L^{p}(\mathbf{B})} & \leq C\left(\sum_{j=0}^{\infty}\left(2^{-j}\right)^{(a-\beta) q^{\prime}}\right)^{1 / q^{\prime}}\left(\sum_{j=0}^{\infty}\left(2^{-j \beta}\||\nabla u|\|_{L^{p}\left(A\left(2^{-j}\right)\right)}\right)^{q}\right)^{1 / q} \\
& \leq C\left(\int_{0}^{1}\left(s^{\beta}\||\nabla u|\|_{L^{p}(A(s))}\right)^{q} \frac{d s}{s}\right)^{1 / q} .
\end{aligned}
$$

This is also true when $0<q \leq 1$. The proof is now completed.

Let us begin with Hardy type inequality in central Herz-Morrey space, as an extension of (1.1).

Theorem 2.2. Let $p \geq 1$ and $\beta<1 / p^{\prime}$. Then there exists a constant $C>0$ such that

$$
\|u\|_{H^{p, q,-1+\beta}(\mathbf{B})} \leq C\|\mid \nabla u\|_{H^{p, q, \beta}(\mathbf{B})}
$$

for $u \in C^{1}\left(\mathbf{R}^{N}\right)$ such that $u=0$ outside $\mathbf{B}$.

Proof. Let $u \in C^{1}\left(\mathbf{R}^{N}\right)$ be a function such that $u=0$ outside $\mathbf{B}$ and $\||\nabla u|\|_{H^{p, q, \beta}(\mathbf{B})}$ $\leq 1$. We show only the case when $p>1$ and $1<q<\infty$, as before. By (2.1), Hölder's 
inequality gives

$$
\begin{aligned}
|u(x)| & \leq|x|\left(\int_{1}^{\infty}|\nabla u(r x)|^{p}(1-r|x|)^{a p} d r\right)^{1 / p}\left(\int_{1}^{1 /|x|}(1-r|x|)^{-a p^{\prime}} d r\right)^{1 / p^{\prime}} \\
& \leq|x|\left(\int_{1}^{\infty}|\nabla u(r x)|^{p}(1-r|x|)^{a p} d r\right)^{1 / p}\left(\frac{1}{1-a p^{\prime}} \frac{(1-|x|)^{-a p^{\prime}+1}}{|x|}\right)^{1 / p^{\prime}} \\
& \leq c_{1}|x|^{1 / p}(1-|x|)^{-a+1 / p^{\prime}}\left(\int_{1}^{\infty}|\nabla u(r x)|^{p}(1-r|x|)^{a p} d r\right)^{1 / p}
\end{aligned}
$$

when $1-a p^{\prime}>0$, where $c_{1}=\left(1-a p^{\prime}\right)^{-1 / p^{\prime}}$. Hence for $0<t<1 / 4$ we obtain by Fubini's theorem

$$
\begin{aligned}
\|u\|_{L^{p}(A(t))} & \leq C t^{-a+1 / p^{\prime}}\left(\int_{1}^{\infty}\left(\int_{A(t)}|\nabla u(r x)|^{p}(1-r|x|)^{a p} d x\right) d r\right)^{1 / p} \\
& \leq C t^{-a+1 / p^{\prime}}\left(\int_{1}^{\infty}\left(\int_{\{y \in \mathbf{B}: y / r \in A(t)\}}|\nabla u(y)|^{p}(1-|y|)^{a p} r^{-1-N} d y\right) d r\right)^{1 / p} \\
& \leq C t^{-a+1 / p^{\prime}}\left(\int_{\mathbf{B} \backslash B(0,1-2 t)}|\nabla u(y)|^{p}(1-|y|)^{a p}\left(\int_{|y| /(1-t)}^{|y| /(1-2 t)} r^{-1-N} d r\right) d y\right)^{1 / p} \\
& \leq C t^{-a+1 / p^{\prime}}\left(\int_{\mathbf{B} \backslash B(0,1-2 t)}|\nabla u(y)|^{p}(1-|y|)^{a p}(t /|y|) d y\right)^{1 / p} \\
& \leq C t^{-a+1}\left(\int_{\mathbf{B} \backslash B(0,1-2 t)}|\nabla u(y)|^{p}(1-|y|)^{a p} d y\right)^{1 / p} \\
& \leq C t^{-a+1} \sum_{j=0}^{\infty}\left(2^{-j} t\right)^{a}\||\nabla u|\|_{L^{p}\left(A\left(2^{-j} t\right)\right) \cdot}
\end{aligned}
$$

For $0<\varepsilon<a-\beta$, we obtain by Hölder's inequality

$$
\begin{aligned}
& \|u\|_{L^{p}(A(t))} \\
& \leq C t^{-a+1}\left(\sum_{j=0}^{\infty}\left(2^{-j} t\right)^{(a-\varepsilon-\beta) q^{\prime}}\right)^{1 / q^{\prime}}\left(\sum_{j=0}^{\infty}\left(\left(2^{-j} t\right)^{\beta+\varepsilon}\||\nabla u|\|_{L^{p}\left(A\left(2^{-j} t\right)\right)}\right)^{q}\right)^{1 / q} \\
& \leq C t^{1-(\varepsilon+\beta)}\left(\int_{0}^{t}\left(s^{\beta+\varepsilon}\|\mid \nabla u\|_{L^{p}(A(s))}\right)^{q} \frac{d s}{s}\right)^{1 / q} .
\end{aligned}
$$

Consequently,

$$
\begin{aligned}
\int_{0}^{1 / 4}\left(t^{-1+\beta}\|u\|_{L^{p}(A(t))}\right)^{q} \frac{d t}{t} & \leq C \int_{0}^{1 / 4} t^{-\varepsilon q}\left(\int_{0}^{t}\left(s^{\beta+\varepsilon}\||\nabla u|\|_{L^{p}(A(s))}\right)^{q} \frac{d s}{s}\right) \frac{d t}{t} \\
& \leq C \int_{0}^{1 / 4}\left(s^{\beta+\varepsilon}\||\nabla u|\|_{L^{p}(A(s))}\right)^{q}\left(\int_{s}^{1 / 4} t^{-\varepsilon q} \frac{d t}{t}\right) \frac{d s}{s} \\
& \leq C \int_{0}^{1 / 4}\left(r^{\beta}\||\nabla u|\|_{L^{p}(A(r))}\right)^{q} \frac{d s}{s} .
\end{aligned}
$$


Hardy-Sobolev inequalities for Sobolev functions in central Herz-Morrey spaces on the unit ball 1035

Finally we find from Lemma 2.1

$$
\int_{1 / 4}^{1}\left(t^{-1+\beta}\|u\|_{L^{p}(A(t))}\right)^{q} \frac{d t}{t} \leq C\left(\|u\|_{L^{p}(B(0,3 / 4))}\right)^{q} \int_{1 / 4}^{1} \frac{d t}{t} \leq C,
$$

which completes the proof.

Theorem 2.3. Let $p \geq 1$ and set $\omega(r)=r^{-1 / p}(\log (e / r))^{-\gamma}$ for $0<r<1$, where

$$
\begin{cases}\gamma=1 & \text { when } q \geq p \\ \gamma=1-1 / p+1 / q & \text { when } 0<q<p .\end{cases}
$$

Then there exists a constant $C>0$ such that

$$
\|u\|_{H^{p, q, \omega}(\mathbf{B})} \leq C\|\mid \nabla u\|_{H^{p, q, 1 / p^{\prime}}(\mathbf{B})}
$$

for $u \in C^{1}(\mathbf{B})$ such that $u=0$ on $B(0,1 / 2)$.

Proof. Let $u \in C^{1}(\mathbf{B})$ be a function such that $u=0$ on $B(0,1 / 2)$. Then

$$
u(x)=\int_{0}^{1} \frac{d}{d r} u(r x) d r
$$

so that

$$
|u(x)| \leq \int_{0}^{1}|x||\nabla u(r x)| d r .
$$

By Hölder's inequality, we obtain

$$
\begin{aligned}
|u(x)| \leq & |x|\left(\int_{0}^{1}|\nabla u(r x)|^{p}(\log (1 /(1-r|x|)))^{a p}\{(1-r|x|) /|x|\}^{p / p^{\prime}} d r\right)^{1 / p} \\
& \times\left(\int_{0}^{1}(\log (1 /(1-r|x|)))^{-a p^{\prime}}\{(1-r|x|) /|x|\}^{-1} d r\right)^{1 / p^{\prime}} \\
\leq & C|x|(\log (1 /(1-|x|)))^{-a+1 / p^{\prime}} \\
& \times\left(\int_{0}^{1}|\nabla u(r x)|^{p}(\log (1 /(1-r|x|)))^{a p}\{(1-r|x|) /|x|\}^{p / p^{\prime}} d r\right)^{1 / p}
\end{aligned}
$$

when $0<a<1 / p^{\prime}$. Hence for $0<t<1 / 4$ we obtain by Fubini's theorem

$$
\begin{aligned}
&\|u\|_{L^{p}(A(t))} \leq C(\log (1 / t))^{-a+1 / p^{\prime}} \\
& \quad \times\left(\int_{0}^{1}\left(\int_{A(t)}|x|^{p}|\nabla u(r x)|^{p}(\log (1 /(1-r|x|)))^{a p}\{(1-r|x|) /|x|\}^{p / p^{\prime}} d x\right) d r\right)^{1 / p} \\
& \leq C(\log (1 / t))^{-a+1 / p^{\prime}} \\
& \quad \times\left(\int_{0}^{1}\left(\int_{\{y: y / r \in A(t)\}}|\nabla u(y)|^{p}|y|(\log (1 /(1-|y|)))^{a p}(1-|y|)^{p / p^{\prime}} r^{-1-N} d y\right) d r\right)^{1 / p} \\
& \leq C(\log (1 / t))^{-a+1 / p^{\prime}}\left(\int_{B(0,1-t)}|\nabla u(y)|^{p}|y|(\log (1 /(1-|y|)))^{a p}(1-|y|)^{p / p^{\prime}}\right. \\
&\left.\quad \times\left(\int_{|y| /(1-t)}^{|y| /(1-2 t)} r^{-1-N} d r\right) d y\right)^{1 / p}
\end{aligned}
$$




$$
\begin{aligned}
\leq & C(\log (1 / t))^{-a+1 / p^{\prime}} \\
& \times\left(\int_{B(0,1-t)}|\nabla u(y)|^{p}|y|(\log (1 /(1-|y|)))^{a p}(1-|y|)^{p / p^{\prime}}(t /|y|) d y\right)^{1 / p} \\
\leq & C(\log (1 / t))^{-a+1 / p^{\prime}} t^{1 / p}\left(\int_{B(0,1-t)}|\nabla u(y)|^{p}(\log (1 /(1-|y|)))^{a p}(1-|y|)^{p / p^{\prime}} d y\right)^{1 / p} \\
\leq & \left.C(\log (1 / t))^{-a+1 / p^{\prime}} t^{1 / p}\left(\sum_{j=0}^{\infty}\left(\left(\log \left(1 /\left(2^{j} t\right)\right)\right)\right)^{a}\left(2^{j} t\right)^{1 / p^{\prime}}\||\nabla u|\|_{L^{p}\left(A\left(2^{j} t\right)\right)}\right)^{p}\right)^{1 / p}
\end{aligned}
$$

so that

$$
\begin{aligned}
& \left(t^{-1 / p}(\log (1 / t))^{-1}\|u\|_{L^{p}(A(t))}\right)^{q} \leq C(\log (1 / t))^{\left(-a+1 / p^{\prime}-1\right) q} \\
& \times\left(\sum_{j=0}^{\infty}\left(\left(\log \left(1 /\left(2^{j} t\right)\right)\right)^{a}\left(2^{j} t\right)^{1 / p^{\prime}}\||\nabla u|\|_{L^{p}\left(A\left(2^{j} t\right)\right)}\right)^{p}\right)^{q / p} .
\end{aligned}
$$

If $q<p$, then

$$
\begin{aligned}
& \left(t^{-1 / p}(\log (1 / t))^{-1}\|u\|_{L^{p}(A(t))}\right)^{q} \leq C(\log (1 / t))^{\left(-a+1 / p^{\prime}-1\right) q} \\
& \times \sum_{j=0}^{\infty}\left(\left(\log \left(1 /\left(2^{j} t\right)\right)\right)^{a}\left(2^{j} t\right)^{1 / p^{\prime}}\||\nabla u|\|_{L^{p}\left(A\left(2^{j} t\right)\right)}\right)^{q} \\
& \leq C(\log (1 / t))^{\left(-a+1 / p^{\prime}-1\right) q} \int_{t}^{1}\left((\log (1 / s))^{a} s^{1 / p^{\prime}}\||\nabla u|\|_{L^{p}(A(s))}\right)^{q} s^{-1} d s
\end{aligned}
$$

and hence

$$
\begin{aligned}
& \int_{0}^{1 / 4}\left(t^{-1 / p}(\log (1 / t))^{-1+1 / p-1 / q}\|u\|_{L^{p}(A(t))}\right)^{q} \frac{d t}{t} \\
& \leq C \int_{0}^{1 / 4}(\log (1 / t))^{-a q-1}\left(\int_{t}^{1}\left((\log (1 / s))^{a} s^{1 / p^{\prime}}\||\nabla u|\|_{L^{p}(A(s))}\right)^{q} s^{-1} d s\right) \frac{d t}{t} \\
& \leq C \int_{0}^{1}\left(s^{1 / p^{\prime}}(\log (1 / s))^{a}\||\nabla u|\|_{L^{p}(A(s))}\right)^{q}\left(\int_{0}^{s}(\log (1 / t))^{-a q-1} \frac{d t}{t}\right) \frac{d s}{s} \\
& \leq C \int_{0}^{1}\left(s^{1 / p^{\prime}}\||\nabla u|\|_{L^{p}(A(s))}\right)^{q} \frac{d s}{s} .
\end{aligned}
$$

If $b=q / p \geq 1$ and $0<\varepsilon<1 / b^{\prime}$, then

$$
\begin{aligned}
& \left(t^{-1 / p}(\log (1 / t))^{-1}\|u\|_{L^{p}(A(t))}\right)^{q} \leq C(\log (1 / t))^{\left(-a+1 / p^{\prime}-1\right) q} \\
& \times\left(\int_{t}^{1}\left((\log (1 / s))^{a} s^{1 / p^{\prime}}\||\nabla u|\|_{L^{p}(A(s))}\right)^{p} s^{-1} d s\right)^{q / p} \\
& \leq C(\log (1 / t))^{(-a-1 / p) q}\left(\int_{t}^{1}(\log (1 / s))^{-\varepsilon b^{\prime}} d s\right)^{b / b^{\prime}} \\
& \quad \times \int_{0}^{2 t}\left((\log (1 / s))^{a+\varepsilon b / q} s^{1 / p^{\prime}}\||\nabla u|\|_{L^{p}(A(s))}\right)^{q} s^{-1} d s
\end{aligned}
$$




$$
\leq C(\log (1 / t))^{(-a-1 / p) q+\left(-\varepsilon+1 / b^{\prime}\right) b} \int_{t}^{1}\left((\log (1 / s))^{a+\varepsilon b / q} s^{1 / p^{\prime}}\||\nabla u|\|_{L^{p}(A(s))}\right)^{q} s^{-1} d s
$$

and hence

$$
\begin{aligned}
& \int_{0}^{1 / 4}\left(t^{-1 / p}(\log (1 / t))^{-1}\|u\|_{L^{p}(A(t))}\right)^{q} \frac{d t}{t} \\
& \leq C \int_{0}^{1 / 4}(\log (1 / t))^{-(a+\varepsilon b / q) q-1}\left(\int_{t}^{1}\left((\log (1 / s))^{a+\varepsilon b / q} s^{1 / p^{\prime}}\||\nabla u|\|_{L^{p}(A(s))}\right)^{q} s^{-1} d s\right) \frac{d t}{t} \\
& \leq C \int_{0}^{1}\left(s^{1 / p^{\prime}}(\log (1 / s))^{a+\varepsilon b / q}\||\nabla u|\|_{L^{p}(A(s))}\right)^{q}\left(\int_{0}^{s}(\log (1 / t))^{-(a+\varepsilon b / q) q-1} \frac{d t}{t}\right) \frac{d s}{s} \\
& \leq C \int_{0}^{1}\left(s^{1 / p^{\prime}}\||\nabla u|\|_{L^{p}(A(s))}\right)^{q} \frac{d s}{s} .
\end{aligned}
$$

With the aid of Lemma 2.1, we complete the proof.

\section{Hardy-Sobolev inequality for Sobolev functions}

Our aim in this section is to give Hardy-Sobolev inequalities for Sobolev functions in $C_{0}^{1}(\mathbf{B})$.

Theorem 3.1. Let $p \geq 1$ and $\beta<1 / p^{\prime}$. For $0 \leq \lambda \leq 1$, set $1 / p_{\lambda}=1 / p-\lambda / N$. Then there exists a constant $C>0$ such that

$$
\|u\|_{H^{p_{\lambda}, q, \lambda-1+\beta}(\mathbf{B})} \leq C\||\nabla u|\|_{H^{p, q, \beta}(\mathbf{B})}
$$

for $u \in C_{0}^{1}(\mathbf{B})$.

When $q=p$ and $\lambda=1$, this is the weighted Sobolev's inequality (see Corollary 3.5 below). For a proof of Theorem 3.1, we first give the classical Sobolev's inequality and then apply the methods expanded in Theorem 2.2.

Now we prepare the following two lemmas.

Lemma 3.2. (Sobolev's inequality, see e.g. $[1,13,14]$ ) Let $p \geq 1$ and $1 / p^{*}=$ $1 / p-1 / N>0$. Then there is a constant $C>0$ such that

$$
\|v\|_{L^{p^{*}}\left(\mathbf{R}^{N}\right)} \leq C\|\mid \nabla v\|_{L^{p}\left(\mathbf{R}^{N}\right)}
$$

for $v \in C_{0}^{1}\left(\mathbf{R}^{N}\right)$.

Let $\tilde{A}(r)=\{y \in \mathbf{B}: r / 2 \leq 1-|y|<4 r\}$.

Lemma 3.3. Let $p \geq 1$ and $1 / p^{*}=1 / p-1 / N>0$. Then there is a constant $C>0$ such that

$$
\|u\|_{L^{p^{*}}(A(r))} \leq C\left\{r^{-1}\|u\|_{L^{p}(\tilde{A}(r))}+\||\nabla u|\|_{L^{p}(\tilde{A}(r))}\right\}
$$

for $u \in C_{0}^{1}\left(\mathbf{R}^{N}\right)$ and $r>0$.

For this, take a continuous function $\varphi$ such that $0 \leq \varphi(r) \leq 1$,

$$
\varphi(r)= \begin{cases}1 & \text { when } 1 / 4<r<1 / 2 \\ 0 & \text { when } r<1 / 8 \text { and } r>1\end{cases}
$$

and $\left|\varphi^{\prime}\right| \leq 8$, and apply Lemma 3.2 with $v(x)=\varphi((1-|x|) /(4 r)) u(x)$.

Now we are ready to prove Theorem 3.1. 

1.

Proof of Theorem 3.1. Let $u \in C_{0}^{1}(\mathbf{B})$ be a function such that $\|\mid \nabla u\|_{H^{p, q, \beta}(\mathbf{B})} \leq$

First we treat the case $\lambda=1$; in this case, $p_{\lambda}$ is the Sobolev exponent $p^{*}$. We show

$$
\|u\|_{H^{p^{*}, q, \beta}(\mathbf{B})} \leq C\|\mid \nabla u\|_{H^{p, q, \beta}(\mathbf{B})} .
$$

In fact, when $1<q<\infty$, we find from Lemma 3.3 and Theorem 2.2

$$
\begin{aligned}
\int_{0}^{1}\left(r^{\beta}\|u\|_{L^{p^{*}}(A(r))}\right) \frac{d r}{r} \leq & C \int_{0}^{1}\left(r^{\beta} r^{-1}\|u\|_{L^{p}(\tilde{A}(r))} \|\right)^{q} \frac{d r}{r} \\
& +C \int_{0}^{1}\left(r^{\beta}\|\mid \nabla u\|_{L^{p}(\tilde{A}(r))}\right)^{q} \frac{d r}{r} \\
\leq & C \int_{0}^{1}\left(r^{\beta}\||\nabla u|\|_{L^{p}(\tilde{A}(r))}\right)^{q} \frac{d r}{r}
\end{aligned}
$$

as required.

Next we treat the case $0 \leq \lambda<1$. By Jensen's inequality we obtain

$$
\|u\|_{L^{p_{1}}(A(r))} \leq|A(r)|^{1 / p_{1}-1 / p_{2}}\|u\|_{L^{p_{2}(A(r))}}
$$

when $p_{2} \geq p_{1} \geq 1$. Therefore

$$
\|u\|_{L^{p_{\lambda}(A(r))}} \leq|A(r)|^{1 / p_{\lambda}-1 / p^{*}}\|u\|_{L^{p^{*}(A(r))}} \leq C r^{-(\lambda-1)}\|u\|_{L^{p^{*}}(A(r))} .
$$

Hence by Lemma 3.2

$$
\begin{aligned}
\int_{0}^{1}\left(r^{\lambda-1+\beta}\|u\|_{\left.L^{p_{\lambda}(A(r))}\right)}\right)^{q} \frac{d r}{r} & \leq C \int_{0}^{1}\left(r^{\beta}\|u\|_{L^{p^{*}}(\tilde{A}(r))}\right)^{q} \frac{d r}{r} \\
& \leq C \int_{0}^{1}\left(r^{\beta}\||\nabla u|\|_{L^{p}(\tilde{A}(r))}\right)^{q} \frac{d r}{r} .
\end{aligned}
$$

This completes the proof.

In the borderline case $\beta=1 / p^{\prime}$, we establish the following result by using Theorem 2.3.

Theorem 3.4. Let $p \geq 1$. For $0 \leq \lambda \leq 1$, set $1 / p_{\lambda}=1 / p-\lambda / N$ and $\omega(r)=$ $r^{\lambda-1+1 / p^{\prime}}(\log (e / r))^{-\gamma}$ for $0<r<1$, where $\gamma$ is the constant appearing in Theorem 2.3. Then there exists a constant $C>0$ such that

$$
\|u\|_{H^{p_{\lambda}, q, \omega}(\mathbf{B})} \leq C\|\mid \nabla u\|_{H^{p, q, 1 / p^{\prime}}(\mathbf{B})}
$$

for all $u \in C_{0}^{1}(\mathbf{B})$ such that $u=0$ on $B(0,1 / 2)$.

When $q=p$, we have the weighted inequalities for Sobolev functions.

Corollary 3.5. Let $p \geq 1, \beta<1 / p^{\prime}, 0 \leq \lambda \leq 1$ and $1 / p_{\lambda}=1 / p-\lambda / N$. Then there exists a constant $C>0$ such that

$$
\int_{\mathbf{B}}|u(x)|^{p_{\lambda}}(1-|x|)^{(\lambda-1+\beta) p_{\lambda}} d x \leq C
$$

for all $u \in C_{0}^{1}(\mathbf{B})$ such that $\||\nabla u|\|_{H^{p, p, \beta}(\mathbf{B})} \leq 1$.

Corollary 3.6. Let $p \geq 1$. Let $0 \leq \lambda \leq 1$ and $1 / p_{\lambda}=1 / p-\lambda / N$. Then

$$
\int_{\mathbf{B}}|u(x)|^{p_{\lambda}}\left((1-|x|)^{\lambda-1+1 / p^{\prime}}(\log (e /(1-|x|)))^{-1}\right)^{p_{\lambda}} d x \leq C
$$

for all $u \in C_{0}^{1}(\mathbf{B})$ such that $u=0$ on $B(0,1 / 2)$ and $\||\nabla u|\|_{H^{p, p, 1 / p^{\prime}}(\mathbf{B})} \leq 1$. 
Hardy-Sobolev inequalities for Sobolev functions in central Herz-Morrey spaces on the unit ball 1039

Our results here give (weighted) Sobolev's inequality when $\lambda=1$, and Hardy's inequality (no account of the best constant) when $\lambda=0$.

\section{Generalized Riesz potentials}

We use the following lemma by polar coordinate. Let $d S$ denote the surface area measure on $\partial \mathbf{B}$.

Lemma 4.1. Let $0<\varepsilon<1$ and $0<r<1$. Then

$$
\int_{\partial B(0, r) \backslash B(x,(1-|x|) / 2)}|x-y|^{\varepsilon-N} d S(y) \leq C(1-|x|)^{\varepsilon-1}
$$

when $r>|x|$ and

$$
\int_{\partial B(0, r) \backslash B(x,(1-|x|) / 2)}|x-y|^{\varepsilon-N} d S(y) \leq C(1-r)^{\varepsilon-1}
$$

when $r \leq|x|$.

Lemma 4.2. There exists a constant $C>0$ such that

$$
\left(\int_{\mathbf{B}}|f(y)|^{p}(1-|y|)^{\beta p} d y\right)^{1 / p} \leq C \int_{0}^{1}\|f\|_{L^{p}(A(r))} r^{\beta} r^{-1} d r .
$$

Proof.

$$
\int_{\mathbf{B}}|f(y)|^{p}(1-|y|)^{\beta p} d y \leq \sum_{j=0}^{\infty} \int_{A\left(2^{-j}\right)}|f(y)|^{p}(1-|y|)^{\beta p} d y
$$

so that

$$
\begin{aligned}
\left(\int_{\mathbf{B}}|f(y)|^{p}(1-|y|)^{\beta p} d y\right)^{1 / p} & \leq \sum_{j=0}^{\infty}\left(\int_{A\left(2^{-j}\right)}|f(y)|^{p}(1-|y|)^{\beta p} d y\right)^{1 / p} \\
& \leq C \sum_{j=0}^{\infty}\|f\|_{L^{p}\left(A\left(2^{-j}\right)\right)} 2^{-j \beta}
\end{aligned}
$$

If $2^{-j} \leq r<2^{-j+1}<2 r \leq 2^{-j+2}$, then

$$
\|f\|_{L^{p}\left(A\left(2^{-j}\right)\right)} \leq\|f\|_{L^{p}(A(r / 2,2 r))} \leq\|f\|_{L^{p}(A(r / 2))}+\|f\|_{L^{p}(A(r))},
$$

where $A(s, t)=\{y \in \mathbf{B}: s \leq 1-|y|<t\}$. Thus

$$
\begin{aligned}
\left(\int_{\mathbf{B}}|f(y)|^{p}(1-|y|)^{\beta p} d y\right)^{1 / p} & \leq C \sum_{j=0}^{\infty} \int_{2^{-j}}^{2^{-j+1}}\|f\|_{L^{p}\left(A\left(2^{-j}\right)\right)} r^{\beta} r^{-1} d r \\
& \leq C \sum_{j=0}^{\infty} \int_{2^{-j}}^{2^{-j+1}}\left(\|f\|_{L^{p}(A(r / 2))}+\|f\|_{L^{p}(A(r))}\right) r^{\beta} r^{-1} d r \\
& \leq C \int_{0}^{1}\|f\|_{L^{p}(A(r))} r^{\beta} r^{-1} d r
\end{aligned}
$$

as required. 
Lemma 4.3. [18, Lemma 2.1] Let $x, y \in \mathbf{R}^{N}$ and $t \in \mathbf{R}$. If $|t|\left|y^{*}\right|<(\sqrt{2}-1) \mid x-$ $y^{*}$, then

$$
\begin{aligned}
\left|x-y^{*}+t y^{*}\right|^{\alpha-N} & =\sum_{\ell=0}^{\infty}\left(\sum_{\ell / 2 \leq j \leq \ell} a_{\ell, j}\left|x-y^{*}\right|^{\alpha-N-2 j}\left(x \cdot y^{*}-\left|y^{*}\right|^{2}\right)^{2 j-\ell}\left|y^{*}\right|^{2(\ell-j)}\right) t^{\ell} \\
& =\sum_{\ell=0}^{\infty} \phi_{\alpha, \ell}\left(x, y^{*}\right) t^{\ell}
\end{aligned}
$$

where

$$
\phi_{\alpha, \ell}\left(x, y^{*}\right)=\sum_{\ell / 2 \leq j \leq \ell} a_{\ell, j}\left|x-y^{*}\right|^{\alpha-N-2 j}\left(x \cdot y^{*}-\left|y^{*}\right|^{2}\right)^{2 j-\ell}\left|y^{*}\right|^{2(\ell-j)}
$$

and

$$
a_{\ell, j}=\left(\begin{array}{c}
(\alpha-N) / 2 \\
j
\end{array}\right)\left(\begin{array}{c}
j \\
\ell-j
\end{array}\right) 2^{2 j-\ell}
$$

For this, write

$$
|x-y|^{2}=\left|x-y^{*}+t y^{*}\right|^{2}=\left|x-y^{*}\right|^{2}\left(1+s /\left|x-y^{*}\right|^{2}\right),
$$

where $t=1-|y|^{2}, y^{*}=y /|y|^{2}$ and $s=t^{2}\left|y^{*}\right|^{2}+2 t\left(x-y^{*}\right) \cdot y^{*}$. Note that

$$
(1+a+b)^{\gamma}=\sum_{j=0}^{\infty}\left(\begin{array}{l}
\gamma \\
j
\end{array}\right)(a+b)^{j}=\sum_{j=0}^{\infty} \sum_{k=0}^{j}\left(\begin{array}{l}
\gamma \\
j
\end{array}\right)\left(\begin{array}{l}
j \\
k
\end{array}\right) a^{k} b^{j-k}
$$

The double series converges absolutely when $|a|+|b|<1$, which proves the lemma.

For $m \geq 0$ let us define

$$
K_{\alpha, m}(x, y)=c(\alpha, N) \times\left\{\begin{array}{lc}
|x-y|^{\alpha-N} & \text { when } y \in B(0,1 / 2), \\
|x-y|^{\alpha-N}-\sum_{\ell=0}^{m}\left(1-|y|^{2}\right)^{\ell} \phi_{\alpha, \ell}\left(x, y^{*}\right) & \text { when } y \in \mathbf{B} \backslash B(0,1 / 2),
\end{array}\right.
$$

where $c(\alpha, N)=1 /\left((N-\alpha) \omega_{N-1}\right)$; set $K_{\alpha,-1}(x, y)=c(\alpha, N)|x-y|^{\alpha-N}$.

Lemma 4.4. [18, Lemma 2.2]

(1) For $y \in \mathbf{B}$ and $N>2, \Delta K_{2, m}(\cdot, y)=\beta_{y}$ on $\mathbf{B}$;

(2) there exists a constant $C>0$ such that

$$
\left|K_{\alpha, m}(x, y)\right| \leq C|x-y|^{\alpha-N}
$$

for all $x, y \in \mathbf{B}$;

(3) there exists a constant $C>0$ such that

$$
\left|K_{\alpha, m}(x, y)\right| \leq C|x-y|^{\alpha-N-m-1}(1-|y|)^{m+1}
$$

for all $x, y \in \mathbf{B}$.

We know the classical Sobolev inequality for Riesz potentials of $L^{p}$-functions (see, e.g. [1, Theorem 3.1.4 (b)], [14]).

Lemma 4.5. (Sobolev's inequality) Let $1 / p_{\alpha}=1 / p-\alpha / N>0$. Then

$$
\left\|I_{\alpha} f\right\|_{L^{p_{\alpha}\left(\mathbf{R}^{N}\right)}} \leq C\|f\|_{L^{p}\left(\mathbf{R}^{N}\right)} .
$$


Hardy-Sobolev inequalities for Sobolev functions in central Herz-Morrey spaces on the unit ball 1041

For $x \in \mathbf{B}$, set

$$
\begin{aligned}
& E_{1}=B(x,(1-|x|) / 2), \\
& E_{2}=\{x \in \mathbf{B} \backslash B(x,(1-|x|) / 2): 1-|y|<1-|x|\} \\
& E_{3}=\{x \in \mathbf{B} \backslash B(x,(1-|x|) / 2): 1-|y| \geq 1-|x|\} .
\end{aligned}
$$

For a nonnegative measurable function $f$ on $\mathbf{B}$, write

$$
I_{j}(x)=\int_{E_{j}} K_{\alpha, m}(x, y) f(y) d y, \quad j=1,2,3
$$

and

$$
I_{\alpha, m} f(x)=I_{1}(x)+I_{2}(x)+I_{3}(x) .
$$

For $0 \leq \lambda \leq \alpha$, set $1 / p_{\lambda}=1 / p-\lambda / N$.

Lemma 4.6. Suppose $f \in L_{\mathrm{loc}}^{1}(\mathbf{B})$. Then

$$
\left\|I_{1}\right\|_{H^{p_{\lambda}, q, \lambda-\alpha+\beta}(\mathbf{B})} \leq C\|f\|_{H^{p, q, \beta}(\mathbf{B})} .
$$

Proof. By Jensen's inequality we obtain

$$
\left\|I_{\alpha} f\right\|_{L^{p_{1}(A(r))}} \leq|A(r)|^{1 / p_{1}-1 / p_{2}}\left\|I_{\alpha} f\right\|_{L^{p_{2}}(A(r))}
$$

when $p_{2} \geq p_{1} \geq 1$. Therefore

$$
\left\|I_{\alpha} f\right\|_{L^{p_{\lambda}}(A(r))} \leq|A(r)|^{1 / p_{\lambda}-1 / p_{\alpha}}\left\|I_{\alpha} f\right\|_{L^{p_{\alpha}(A(r))}} \leq C r^{-(\lambda-\alpha)}\left\|I_{\alpha} f\right\|_{L^{p_{\alpha}}(A(r))} .
$$

Hence by Lemma 4.5

$$
\begin{aligned}
\int_{0}^{1}\left(t^{\lambda-\alpha+\beta}\left\|I_{1}\right\|_{\left.L^{p_{\lambda}(A(t))}\right)^{q} d t / t}\right. & \leq C \int_{0}^{1}\left(t^{\lambda-\alpha+\beta}\left\|I_{\alpha} f\right\|_{L^{p_{\lambda}(A(t))}}\right)^{q} d t / t \\
& \leq C \int_{0}^{1}\left(t^{\beta}\left\|I_{\alpha} f\right\|_{L^{p_{\alpha}(A(t))}}\right)^{q} d t / t \\
& \leq C \int_{0}^{1}\left(t^{\beta}\|f\|_{L^{p}(A(t))}\right)^{q} d t / t
\end{aligned}
$$

which proves the result.

Lemma 4.7. Suppose $0<\alpha \leq m+2,0 \leq \lambda \leq \alpha$ and $\beta<m+1+1 / p^{\prime}$. Then there exists a constant $C>0$ such that

$$
\left\|I_{2}\right\|_{H^{p_{\lambda}, q, \lambda-\alpha+\beta}(\mathbf{B})} \leq C
$$

when $\|f\|_{H^{p, q, \beta}(\mathbf{B})} \leq 1$.

Proof. Let $f$ be a nonnegative measurable function on $\mathbf{B}$ such that $\|f\|_{H^{p, q, \beta}(\mathbf{B})} \leq$ 1. We treat only the case $1<q<\infty$ for convenience. Taking $\varepsilon>0$ and $\gamma$ such that 
$\beta-m-1+\varepsilon<\gamma<1 / p^{\prime}$, we have by Hölder's inequality and (4.1)

$$
\begin{aligned}
\left|I_{2}(x)\right| \leq & C\left(\int_{E_{2}}|x-y|^{\alpha-N-m-1}(1-|y|)^{-\gamma p^{\prime}} d y\right)^{1 / p^{\prime}} \\
& \times\left(\int_{E_{2}}|x-y|^{\alpha-N-m-1} f(y)^{p}(1-|y|)^{(m+1+\gamma) p} d y\right)^{1 / p} \\
\leq & C\left(\int_{0}^{1-|x|}(1-|x|)^{(\alpha-m-1)-1} s^{-\gamma p^{\prime}} d s\right)^{1 / p^{\prime}} \\
& \times\left(\int_{E_{2}}|x-y|^{\alpha-N-m-1} f(y)^{p}(1-|y|)^{(m+1+\gamma) p} d y\right)^{1 / p} \\
\leq & C r^{\left(\alpha-m-1-\gamma p^{\prime}\right) / p^{\prime}}\left(\int_{E_{2}}|x-y|^{\alpha-N-m-1} f(y)^{p}(1-|y|)^{(m+1+\gamma) p} d y\right)^{1 / p}
\end{aligned}
$$

for $x \in A(r)$, so that Minkowski's inequality, Lemma 4.2 and Hölder's inequality give

$$
\begin{aligned}
& \left\|I_{2}\right\|_{L^{p_{\lambda}(A(r))} \leq C r^{\left(\alpha-m-1-\gamma p^{\prime}\right) / p^{\prime}}} \\
& \times\left(\int_{E_{2}} f(y)^{p}(1-|y|)^{(m+1+\gamma) p}\left(\int_{A(r)}|x-y|^{(\alpha-N-m-1) p_{\lambda} / p} d x\right)^{p / p_{\lambda}} d y\right)^{1 / p} \\
& \leq C r^{\left(\alpha-m-1-\gamma p^{\prime}\right) / p^{\prime}}\left(\int_{E_{2}} f(y)^{p}(1-|y|)^{(m+1+\gamma) p} d y\right)^{1 / p} r^{(\alpha-N-m-1) / p+N / p_{\lambda}} \\
& \leq C r^{\alpha-m-1-\lambda-\gamma} \int_{0}^{1}\|f\|_{L^{p}\left(E_{2} \cap A(t)\right)} t^{m+1+\gamma} t^{-1} d t \\
& \leq C r^{\alpha-m-1-\lambda-\gamma}\left(\int_{0}^{2 r} t^{\varepsilon q^{\prime}} t^{-1} d t\right)^{1 / q^{\prime}}\left(\int_{0}^{2 r}\left(t^{-\varepsilon+\gamma+m+1}\|f\|_{L^{p}(A(t))}\right)^{q} t^{-1} d t\right)^{1 / q} \\
& \leq C r^{\alpha-m-1-\lambda-\gamma+\varepsilon}\left(\int_{0}^{2 r}\left(t^{-\varepsilon+\gamma+m+1}\|f\|_{L^{p}(A(t))}\right)^{q} t^{-1} d t\right)^{1 / q} .
\end{aligned}
$$

Hence

$$
\begin{aligned}
& \int_{0}^{1}\left(r^{\lambda-\alpha+\beta}\left\|I_{2}\right\|_{L^{p_{\lambda}(A(r))}}\right)^{q} r^{-1} d r \\
& \leq C \int_{0}^{1}\left(t^{-\varepsilon+\gamma+m+1}\|f\|_{L^{p}(A(t))}\right)^{q}\left(\int_{t / 2}^{1} r^{(-m-1+\beta-\gamma+\varepsilon) q} r^{-1} d r\right) t^{-1} d t \\
& \leq C \int_{0}^{1}\left(t^{\beta}\|f\|_{L^{p}(A(t)}\right)^{q} t^{-1} d t,
\end{aligned}
$$

since $-m-1+\beta-\gamma+\varepsilon<0$.

Lemma 4.8. Suppose $0<\alpha \leq m+2,0 \leq \lambda \leq \alpha$ and $\alpha-1 / p<\beta+\lambda(N-1) / N$. Then there exists a constant $C>0$ such that

$$
\left\|I_{3}\right\|_{H^{p_{\lambda}, q, \lambda-\alpha+\beta}(\mathbf{B})} \leq C
$$

when $\|f\|_{H^{p, q, \beta}(\mathbf{B})} \leq 1$.

Proof. Let $f$ be a nonnegative measurable function on $\mathbf{B}$ such that $\|f\|_{H^{p, q, \beta}(\mathbf{B})} \leq$ 1. We treat only the case $1<q<\infty$ for convenience. Taking $\varepsilon>0$ and $\gamma$ such that 
Hardy-Sobolev inequalities for Sobolev functions in central Herz-Morrey spaces on the unit ball 1043 $(\alpha-m-1) / p^{\prime}<\gamma<\beta-\varepsilon-\alpha / p+1 / p-(m+1) / p^{\prime}+\lambda(N-1) / N$, we have by Hölder's inequality and (4.2)

$$
\begin{aligned}
\left|I_{3}(x)\right| \leq & \left(\int_{E_{3}}|x-y|^{\alpha-N-m-1}(1-|y|)^{-\gamma p^{\prime}} d y\right)^{1 / p^{\prime}} \\
& \times\left(\int_{E_{3}}|x-y|^{\alpha-N-m-1} f(y)^{p}(1-|y|)^{(\gamma+m+1) p} d y\right)^{1 / p} \\
\leq & C\left(\int_{1-|x|}^{1} s^{(\alpha-m-1)-1-\gamma p^{\prime}} d s\right)^{1 / p^{\prime}} \\
& \times\left(\int_{E_{3}}|x-y|^{\alpha-N-m-1} f(y)^{p}(1-|y|)^{(\gamma+m+1) p} d y\right)^{1 / p} \\
\leq & C r^{\left(\alpha-m-1-\gamma p^{\prime}\right) / p^{\prime}}\left(\int_{E_{3}}|x-y|^{\alpha-N-m-1} f(y)^{p}(1-|y|)^{(\gamma+m+1) p} d y\right)^{1 / p}
\end{aligned}
$$

since $\alpha-m-1-\gamma p^{\prime}<0$. By Minkowski's inequality and (4.1), we obtain

$$
\begin{aligned}
& \left\|I_{3}\right\|_{L^{p_{\lambda}(A(r)}} \leq C r^{\left(\alpha-m-1-\gamma p^{\prime}\right) / p^{\prime}} \\
& \times\left(\int_{E_{3}} f(y)^{p}(1-|y|)^{(\gamma+m+1) p}\left(\int_{A(r)}|x-y|^{(\alpha-N-m-1) p_{\lambda} / p} d x\right)^{p / p_{\lambda}} d y\right)^{1 / p} \\
& \leq C r^{\left(\alpha-m-1-\gamma p^{\prime}\right) / p^{\prime}} \\
& \quad \times\left(\int_{E_{3}} f(y)^{p}(1-|y|)^{(\gamma+m+1) p}(1-|y|)^{(\alpha-N-m-1)+(N-1) p / p_{\lambda}} r^{p / p_{\lambda}} d y\right)^{1 / p},
\end{aligned}
$$

since $(\alpha-N-m-1) p_{\lambda} / p+N-1 \leq(N-1)\left(1-p_{\lambda} / p\right)<0$ by our assumption. Therefore, in view of Lemma 4.2 and Hölder's inequality, we obtain

$$
\begin{aligned}
& \left\|I_{3}\right\|_{L^{p_{\lambda}(A(r)}} \\
& \leq C r^{\left(\alpha-m-1-\gamma p^{\prime}\right) / p^{\prime}+1 / p_{\lambda}} \int_{0}^{1}\|f\|_{L^{p}\left(E_{3} \cap A(t)\right)} t^{\gamma+m+1+(\alpha-N-m-1) / p+(N-1) / p_{\lambda}} t^{-1} d t \\
& \leq \\
& \quad C r^{\left(\alpha-m-1-\gamma p^{\prime}\right) / p^{\prime}+1 / p_{\lambda}}\left(\int_{r}^{1} t^{-\varepsilon q^{\prime}} t^{-1} d t\right)^{1 / q^{\prime}} \\
& \quad \times\left(\int_{r}^{1}\left(t^{\varepsilon+\gamma+m+1+(\alpha-N-m-1) / p+(N-1) / p_{\lambda}}\|f\|_{L^{p}(A(t))}\right)^{q} t^{-1} d t\right)^{1 / q} \\
& \leq C r^{\left(\alpha-m-1-\gamma p^{\prime}\right) / p^{\prime}+1 / p_{\lambda}-\varepsilon} \\
& \quad \times\left(\int_{r}^{1}\left(t^{\varepsilon+\gamma+m+1+(\alpha-N-m-1) / p+(N-1) / p_{\lambda}}\|f\|_{L^{p}(A(t))}\right)^{q} t^{-1} d t\right)^{1 / q}
\end{aligned}
$$

Hence

$$
\begin{aligned}
& \int_{0}^{1}\left(r^{\lambda-\alpha+\beta}\left\|I_{3}\right\|_{\left.L^{p_{\lambda}(A(r)}\right)^{q} r^{-1} d r}\right. \\
& \leq C \int_{0}^{1}\left(t^{\varepsilon+\gamma+m+1+(\alpha-N-m-1) / p+(N-1) / p_{\lambda}}\|f\|_{L^{p}(A(t)}\right)^{q} \\
& \quad \times\left(\int_{0}^{t} r^{\left\{\beta-(\alpha-m-1) / p-(\gamma+m+1)+\lambda+1 / p_{\lambda}-\varepsilon\right\} q} r^{-1} d r\right) t^{-1} d t
\end{aligned}
$$




$$
\leq C \int_{0}^{1}\left(t^{\beta}\|f\|_{L^{p}(A(t))}\right)^{q} t^{-1} d t
$$

when $\beta-(\alpha-m-1) / p-(\gamma+m+1)+\lambda+1 / p_{\lambda}-\varepsilon>0$.

With the aid of Lemmas 4.6, 4.7 and 4.8, we obtain the following result.

Theorem 4.9. Let $0 \leq \lambda \leq \alpha$ and $1 / p_{\lambda}=1 / p-\lambda / N$. Suppose $0<\alpha \leq m+2$ and $\alpha-1 / p<\beta+\lambda(N-1) / N<m+1+1 / p^{\prime}+\lambda(N-1) / N$. Then

$$
\left\|I_{\alpha, m} f\right\|_{H^{p_{\lambda}, q, \lambda-\alpha+\beta}(\mathbf{B})} \leq C\|f\|_{H^{p, q, \beta}(\mathbf{B})} .
$$

Remark 4.10. Consider the function

$$
u(x)= \begin{cases}(1-|x|)^{a} & \text { when }|x|<1 \\ 0 & \text { when }|x| \geq 1\end{cases}
$$

If $a>0$ and $(a-1) p+1>0$, then the Sobolev integral representation implies that

$$
u(x) \leq C\left(I_{1}|\nabla u|\right)(x)
$$

and Sobolev's inequality gives

$$
\left\|I_{1}|\nabla u|\right\|_{L^{p^{*}\left(\mathbf{R}^{N}\right)}} \leq C\||\nabla u|\|_{L^{p}(\mathbf{B})} .
$$

But, if $(a-1) p+1 \leq 0$, then this does not hold since $\left(I_{1}|\nabla u|\right)(x)=\infty$ for all $x \in \mathbf{R}^{N}$. This suggests us to consider the generalized Riesz kernels. On the other hand, note that

$$
\int_{\mathbf{B}}\left(|\nabla u(x)|(1-|x|)^{\beta}\right)^{p} d x<\infty
$$

when $(a-1+\beta) p+1>0$. Moreover,

$$
\int_{\mathbf{B}}\left|K_{1, m}(x, y)\right||\nabla u(y)| d y \in L_{\mathrm{loc}}^{1}(\mathbf{B})
$$

for $m$ such that $0<a-1+\beta+1 / p<a-1+(m+1)+1$.

But, as in [18, Remark 2.4], we see that

$$
K_{1,0}(x, y)=|x-y|^{1-N}-\left|x-y^{*}\right|^{1-N} \geq\left|x-y^{*}\right|^{1-N}\left(|y|^{1-N}-1\right),
$$

so that

$$
\int_{\mathbf{B}}\left|K_{1,0}(x, y)\right||\nabla u(y)| d y \geq C \int_{\mathbf{B}}(1-|y|)^{1+a-1} d y=\infty
$$

when $a+1 \leq 0$, and hence Theorem 4.9 does not hold when $\beta>(m+1)+1 / p^{\prime}$ (with $m=0$ ); see also Corollary 4.15 below.

By Theorem 4.9, we obtain the following Hardy and Sobolev type inequalities.

Corollary 4.11. (Hardy type inequality) Suppose $0<\alpha<1$ and $\alpha-1 / p<$ $\beta<1 / p^{\prime}$. If $\|f\|_{H^{p, p, \beta}(\mathbf{B})}<\infty$, then

$$
\int_{\mathbf{B}}\left|I_{\alpha} f(x)\right|^{p}(1-|x|)^{(-\alpha+\beta) p} d x<\infty .
$$

Corollary 4.12. (Sobolev type inequality) Let $0<\alpha<1$ and $1 / p_{\alpha}=1 / p-\alpha / N$. Suppose $\alpha-1 / p<\beta+\alpha(N-1) / N<1 / p^{\prime}+\alpha(N-1) / N$. If $\|f\|_{H^{p, p, \beta}(\mathbf{B})}<\infty$, then

$$
\int_{\mathbf{B}}\left|I_{\alpha} f(x)\right|^{p_{\alpha}}(1-|x|)^{\beta p_{\alpha}} d x<\infty .
$$


Hardy-Sobolev inequalities for Sobolev functions in central Herz-Morrey spaces on the unit ball 1045

We next consider the borderline case. For this, set $\omega(r)=r^{\lambda-\alpha+\beta}(\log (e / r))^{-\gamma}$ for $0<r<1$, where

$$
\begin{cases}\gamma=1 & \text { when } q \geq p \\ \gamma=1-1 / p+1 / q & \text { when } 0<q<p .\end{cases}
$$

Theorem 4.13. Let $1 / p_{\lambda}=1 / p-\lambda / N$. Suppose $0<\alpha<1,0 \leq \lambda \leq \alpha$ and $\alpha-1 / p=\beta+\lambda(N-1) / N<m+1+1 / p^{\prime}+\lambda(N-1) / N$. Then

$$
\left\|I_{\alpha, m} f\right\|_{H^{p}, q, \omega}(\mathbf{B}) \leq C\|f\|_{H^{p, q, \beta}(\mathbf{B})} .
$$

To show this, it suffices to prepare the following result instead of Lemma 4.8.

Lemma 4.14. Let $p \geq 1$. For $0 \leq \lambda \leq \alpha$, set $1 / p_{\lambda}=1 / p-\lambda / N$ and $\omega(r)=$ $r^{\lambda-\alpha+\beta}(\log (e / r))^{-\gamma}$ for $0<r<1$, where

$$
\begin{cases}\gamma=1 & \text { when } q \geq p \\ \gamma=1-1 / p+1 / q & \text { when } 0<q<p .\end{cases}
$$

Suppose $0<\alpha<m+2$ and $\alpha-1 / p=\beta+\lambda(N-1) / N$. Then there exists a constant $C>0$ such that

$$
\left\|I_{3}\right\|_{H^{p_{\lambda}, q, \omega}(\mathbf{B})} \leq C
$$

when $\|f\|_{H^{p, q, \beta}(\mathbf{B})} \leq 1$.

Proof. Let $f$ be a nonnegative measurable function on $\mathbf{B}$ such that $\|f\|_{H^{p, q, \beta}(\mathbf{B})} \leq$ 1. We treat only the case $1<q<p$ for convenience. Taking $0<\varepsilon<1 / p^{\prime}$ and $\gamma$ such that $\gamma=(\alpha-m-1) / p^{\prime}$, we have by Hölder's inequality and (4.2)

$$
\begin{aligned}
\left|I_{3}(x)\right| \leq & \left(\int_{E_{3}}|x-y|^{\alpha-N-m-1}(1-|y|)^{-\gamma p^{\prime}}\left(\log (e /(1-|y|))^{-\varepsilon p^{\prime}} d y\right)^{1 / p^{\prime}}\right. \\
& \times\left(\int_{E_{3}}|x-y|^{\alpha-N-m-1} f(y)^{p}(1-|y|)^{(\gamma+m+1) p}\left(\log (e /(1-|y|))^{\varepsilon p} d y\right)^{1 / p}\right. \\
\leq & C\left(\int_{1-|x|}^{1} s^{(\alpha-m-1)-1-\gamma p^{\prime}}(\log (e / s))^{-\varepsilon p^{\prime}} d s\right)^{1 / p^{\prime}} \\
& \times\left(\int_{E_{3}}|x-y|^{\alpha-N-m-1} f(y)^{p}(1-|y|)^{(\gamma+m+1) p}(\log (e /(1-|y|)))^{\varepsilon p} d y\right)^{1 / p} \\
\leq & C \log (1 /(1-|x|))^{-\varepsilon+1 / p^{\prime}} \\
& \times\left(\int_{E_{3}}|x-y|^{\alpha-N-m-1} f(y)^{p}(1-|y|)^{(\gamma+m+1) p}(\log (e /(1-|y|)))^{\varepsilon p} d y\right)^{1 / p} .
\end{aligned}
$$

Therefore, Minkowski's inequality, (4.1) and Lemma 4.2 give

$$
\begin{aligned}
& \left\|I_{3}\right\|_{L^{p_{\lambda}(A(r))}}^{q} \leq C(\log (e / r))^{\left(-\varepsilon+1 / p^{\prime}\right) q}\left(\int_{E_{3}} f(y)^{p}(1-|y|)^{(\gamma+m+1) p}(\log (e /(1-|y|)))^{\varepsilon p}\right. \\
& \left.\quad \times\left(\int_{A(r)}|x-y|^{(\alpha-N-m-1) p_{\lambda} / p} d x\right)^{p / p_{\lambda}} d y\right)^{q / p}
\end{aligned}
$$




$$
\begin{aligned}
\leq & C(\log (e / r))^{\left(-\varepsilon+1 / p^{\prime}\right) q}\left(\int_{E_{3}} f(y)^{p}(1-|y|)^{(\gamma+m+1) p}\right. \\
& \times(1-|y|)^{(\alpha-N-m-1)+(N-1) p / p_{\lambda}}\left(\log (e /(1-|y|))^{\varepsilon p} r^{p / p_{\lambda}} d y\right)^{q / p} \\
\leq & C(\log (e / r))^{\left(-\varepsilon+1 / p^{\prime}\right) q} r^{q / p_{\lambda}} \int_{0}^{1}\left(\|f\|_{L^{p}\left(E_{3} \cap A(t)\right)} t^{\beta}(\log (e / t))^{\varepsilon}\right)^{q} t^{-1} d t
\end{aligned}
$$

since

$$
\gamma+m+1+(\alpha-N-m-1) / p+(N-1) / p_{\lambda}=\beta
$$

by our assumption. Hence

$$
\begin{aligned}
& \int_{0}^{1}\left(r^{\lambda-\alpha+\beta}(\log (e / r))^{-1+1 / p-1 / q}\left\|I_{3}\right\|_{L^{p_{\lambda}}(A(r))}\right)^{q} r^{-1} d r \\
& \leq C \int_{0}^{1}\left((\log (e / t))^{\varepsilon} t^{\beta}\|f\|_{L^{p}(A(t))}\right)^{q}\left(\int_{0}^{t}(\log (e / r))^{-\varepsilon q-1} r^{-1} d r\right) t^{-1} d t \\
& \leq C \int_{0}^{1}\left(t^{\beta}\|f\|_{L^{p}(A(t))}\right)^{q} t^{-1} d t,
\end{aligned}
$$

which completes the proof.

Let $G(x, y)$ be a Green kernel on $\mathbf{B}(N \geq 3)$. We define the Green potential for $f \in L_{\text {loc }}^{1}(\mathbf{B})$ by

$$
G f(x)=\int_{\mathbf{B}} G(x, y) f(y) d y .
$$

We note that $G f(x) \leq I_{2,0} f(x)$, since $K_{2,0}(x, y)=G(x, y)+\left(|y|^{2-N}-1\right)\left|x-y^{*}\right|^{2-N}$ by [18, Remark 2.4], where $N \geq 3$ and $y^{*}$ denotes the inversion of $y$.

By Theorem 4.9 with $\alpha=2$ and $m=0$, we have the following corollary.

Corollary 4.15. Let $0 \leq \lambda \leq 2<N$ and $1 / p_{\lambda}=1 / p-\lambda / N$. Suppose $2-1 / p<$ $\beta+\lambda(N-1) / N<1+1 / p^{\prime}+\lambda(N-1) / N$. Then

$$
\|G f\|_{H^{p_{\lambda}, q, \lambda-2+\beta}(\mathbf{B})} \leq C\|f\|_{H^{p, q, \beta}(\mathbf{B})} .
$$

Remark 4.16. If $N=2$, then we have to modify Lemma 4.4. In fact, we find for $f \geq 0$

$$
\begin{aligned}
G f(x) \leq & C \int_{E_{1}} f(y) \log (e /|x-y|) d y+C \int_{\mathbf{B} \backslash E_{1}} f(y)(1-|x|)(1-|y|)|x-y|^{-2} d y \\
\leq & C \int_{E_{1}} f(y)|x-y|^{\lambda-2} d y+C \int_{E_{2}} f(y)|x-y|^{-1}(1-|y|) d y \\
& +C \int_{E_{3}} f(y)|x-y|^{-2}(1-|y|)^{2} d y \\
= & C\left\{J_{1}(x)+J_{2}(x)+J_{3}(x)\right\}
\end{aligned}
$$

when $0 \leq \lambda<2$. Suppose $1 / p_{\lambda}=1 / p-\lambda / 2>0,2-1 / p<\beta+\lambda(N-1) / N<$ $1+1 / p^{\prime}+\lambda(N-1) / N$. Then $J_{1}, J_{2}$ and $J_{3}$ are treated by Lemma 4.6 , Lemma 4.7 with $m=0$ and Lemma 4.8 with $m=1$, respectively. Thus

$$
\|G f\|_{H^{p_{\lambda}, q, \lambda-2+\beta}(\mathbf{B})} \leq C\|f\|_{H^{p, q, \beta}(\mathbf{B})} .
$$


Hardy-Sobolev inequalities for Sobolev functions in central Herz-Morrey spaces on the unit ball 1047

\section{Appendix}

For reader's convenience, we give a proof of the classical Hardy inequalities by the fundamental theorem of calculus and Hölder's inequality.

\subsection{Hardy type inequalities on the unit ball.}

Theorem 5.1. Let $p \geq 1$ and $\beta<p-1$. If $u \in C^{1}\left(\mathbf{R}^{N}\right)$ is a function such that $u=0$ outside $\mathbf{B}$, then

$$
\int_{\mathbf{B}}|u(x)|^{p}(1-|x|)^{-p+\beta}|x|^{1-N} d x \leq c(p) \int_{\mathbf{B}}|\nabla u(x)|^{p}(1-|x|)^{\beta}|x|^{1-N} d x
$$

where $c(p)=\left(\frac{p}{p-1-\beta}\right)^{p}$.

Proof. By (2.1) and Hölder's inequality, we have for $\beta / p<a<1 / p^{\prime}$

$$
\begin{aligned}
|u(x)| & \leq|x|\left(\int_{1}^{\infty}|\nabla u(r x)|^{p}(1-r|x|)^{a p} d r\right)^{1 / p}\left(\int_{1}^{1 /|x|}(1-r|x|)^{-a p^{\prime}} d r\right)^{1 / p^{\prime}} \\
& \leq|x|\left(\int_{1}^{\infty}|\nabla u(r x)|^{p}(1-r|x|)^{a p} d r\right)^{1 / p}\left(\frac{1}{1-a p^{\prime}} \frac{(1-|x|)^{-a p^{\prime}+1}}{|x|}\right)^{1 / p^{\prime}} \\
& \leq c_{1}|x|^{1 / p}\left(\int_{1}^{\infty}|\nabla u(r x)|^{p}(1-r|x|)^{a p} d r\right)^{1 / p}(1-|x|)^{-a+1 / p^{\prime}}
\end{aligned}
$$

where $c_{1}=\left(1-a p^{\prime}\right)^{-1 / p^{\prime}}$. Hence

$$
\begin{aligned}
& \int_{\mathbf{B}}|u(x)|^{p}(1-|x|)^{-p+\beta}|x|^{1-N} d x \\
& \leq c_{1}^{p} \int_{1}^{\infty}\left(\int_{\mathbf{B}}|x||\nabla u(r x)|^{p}(1-r|x|)^{a p}(1-|x|)^{\left(-a+1 / p^{\prime}-1\right) p+\beta}|x|^{1-N} d x\right) d r \\
& \leq c_{1}^{p} \int_{1}^{\infty}\left(\int_{\mathbf{B}}|y||\nabla u(y)|^{p}(1-|y|)^{a p}(1-|y| / r)^{(-a p+\beta)-1}|y|^{1-N} d y\right) r^{-2} d r \\
& \leq c_{1}^{p} \int_{\mathbf{B}}|y||\nabla u(y)|^{p}(1-|y|)^{a p}|y|^{1-N}\left(\int_{1}^{\infty}(1-|y| / r)^{(-a p+\beta)-1} r^{-2} d r\right) d y \\
& \left.\leq c_{1}^{p} \int_{\mathbf{B}}|y||\nabla u(y)|^{p}(1-|y|)^{a p}|y|^{1-N}\left[\frac{1}{-a p+\beta} \frac{(1-|y| / r)^{-a p+\beta}}{|y|}\right]_{1}^{\infty}\right) d y \\
& \leq c_{1}^{p} \int_{\mathbf{B}}|y||\nabla u(y)|^{p}(1-|y|)^{a p}|y|^{1-N}\left(\frac{1}{a p-\beta} \frac{(1-|y|)^{-a p+\beta}}{|y|}\right) \\
& =c_{1}^{p} c_{2} \int_{\mathbf{B}}|\nabla u(y)|^{p}(1-|y|)^{\beta}|y|^{1-N} d y,
\end{aligned}
$$

where $c_{2}=(a p-\beta)^{-1}$.

Here we see that $c_{1}^{p} c_{2}=\left(\frac{1}{1-a p^{\prime}}\right)^{p / p^{\prime}} \frac{1}{a p-\beta}$ has the minimum $c(p)$ at $a=(1+$ $\beta) /\left(p+p^{\prime}\right)$.

With a slight modification of the above proof, we obtain the following result.

Theorem 5.2. Let $p \geq 1$ and $\beta<p-1$. If $u \in C^{1}\left(\mathbf{R}^{N}\right)$ is a function such that $u=0$ outside $\mathbf{B}$, then

$$
\int_{\mathbf{B}}|u(x)|^{p}(1-|x|)^{-p+\beta} d x \leq c(p) \int_{\mathbf{B}}|\nabla u(x)|^{p}(1-|x|)^{\beta} d x
$$


where $c(p)=\left(\frac{p}{p-1-\beta}\right)^{p}$.

Next we treat the borderline case $\beta=p-1$.

Theorem 5.3. Let $p \geq 1$. If $u \in C^{1}(\mathbf{B})$ is a function such that $u(0)=0$, then $\int_{\mathbf{B}}|u(x)|^{p}(1-|x|)^{-1}|\log (1 /(1-|x|))|^{-p}|x|^{1-N} d x \leq \tilde{c}(p) \int_{\mathbf{B}}|\nabla u(x)|^{p}(1-|x|)^{p-1}|x|^{1-N} d x$, where $\tilde{c}(p)=\left(\frac{p}{p-1}\right)^{p}$.

Proof. Let $u \in C^{1}(\mathbf{B})$ be a function such that $u(0)=0$. By (2.2) and Hölder's inequality, we obtain

$$
\begin{aligned}
|u(x)| \leq & |x|\left(\left.\int_{0}^{1}|\nabla u(r x)|^{p}|| \log (1 /(1-r|x|))\right|^{a p}\{(1-r|x|) /|x|\}^{p / p^{\prime}} d r\right)^{1 / p} \\
& \times\left(\int_{0}^{1}|\log (1 /(1-r|x|))|^{-a p^{\prime}}\{(1-r|x|) /|x|\}^{-1} d r\right)^{1 / p^{\prime}} \\
\leq & |x|\left(\int_{0}^{1}|\nabla u(r x)|^{p}|\log (1 /(1-r|x|))|^{a p}\{(1-r|x|) /|x|\}^{p / p^{\prime}} d r\right)^{1 / p} \\
& \times\left(\left[\frac{1}{-a p^{\prime}+1}|\log (1 /(1-r|x|))|^{-a p^{\prime}+1}\right]_{0}^{1}\right)^{1 / p^{\prime}} \\
\leq & c_{1}|x|^{1 / p}|\log (1 /(1-|x|))|^{-a+1 / p^{\prime}} \\
& \times\left(\int_{0}^{1}|\nabla u(r x)|^{p}|\log (1 /(1-r|x|))|^{a p}(1-r|x|)^{p / p^{\prime}} d r\right)^{1 / p}
\end{aligned}
$$

when $0<a<1 / p^{\prime}$, where $c_{1}=\left(1-a p^{\prime}\right)^{-1 / p^{\prime}}$. Fubini's theorem gives

$$
\begin{aligned}
& \int_{\mathbf{B}}|u(x)|^{p}(1-|x|)^{-1}|\log (1 /(1-|x|))|^{-p}|x|^{1-N} d x \\
& \leq c_{1}^{p} \int_{0}^{1}\left(\int_{\mathbf{B}}|\nabla u(r x)|^{p}|\log (1 /(1-r|x|))|^{a p}(1-r|x|)^{p / p^{\prime}}\right. \\
&\left.\times|x||\log (1 /(1-|x|))|^{\left(-a+1 / p^{\prime}\right) p}(1-|x|)^{-1}|\log (1 /(1-|x|))|^{-p}|x|^{1-N} d x\right) d r \\
&= c_{1}^{p} \int_{0}^{1}\left(r^{-2} \int_{\{y \in \mathbf{B}:|y|<r\}}|\nabla u(y)|^{p}|\log (1 /(1-|y|))|^{a p}(1-|y|)^{p / p^{\prime}}\right. \\
&\left.\times|y||\log (1 /(1-|y| / r))|^{\left(-a+1 / p^{\prime}\right) p}(1-|y| / r)^{-1}|\log (1 /(1-|y| / r))|^{-p}|y|^{1-N} d y\right) d r \\
& \leq c_{1}^{p} \int_{\mathbf{B}}|\nabla u(y)|^{p}|y||\log (1 /(1-|y|))|^{a p}(1-|y|)^{p / p^{\prime}}|y|^{1-N} \\
& \times\left(\int_{|y|}^{1}|\log (1 /(1-|y| / r))|^{-a p-1}(1-|y| / r)^{-1}|y| r^{-2} d r\right) d y \\
& \leq c_{1}^{p} \int_{\mathbf{B}}|\nabla u(y)|^{p}|y||\log (1 /(1-|y|))|^{a p}(1-|y|)^{p / p^{\prime}}|y|^{1-N} \\
& \times\left[\frac{1}{a p}|\log (1 /(1-|y| / r))|^{-a p}\right]_{|y|}^{1} d y
\end{aligned}
$$


Hardy-Sobolev inequalities for Sobolev functions in central Herz-Morrey spaces on the unit ball 1049

$$
\leq c_{1}^{p} c_{3} \int_{\mathbf{B}}|\nabla u(y)|^{p}(1-|y|)^{p / p^{\prime}}|y|^{1-N} d y
$$

where $c_{3}=1 /(a p)$.

Here we see that $c_{1}^{p} c_{3}=\left(\frac{1}{1-a p^{\prime}}\right)^{p / p^{\prime}} \frac{1}{a p}$ has the minimum $\tilde{c}(p)$ at $a=1 /\left(p+p^{\prime}\right)$.

5.2. Hardy type inequalities outside the unit ball. For reader's convenience, we give Hardy type inequalities outside $\mathbf{B}$.

Theorem 5.4. Let $p \geq 1$ and $\beta<p-1$. If $u \in C^{1}\left(\mathbf{R}^{N}\right)$ is a function such that $u=0$ on $\mathbf{B}$, then

$$
\int_{\mathbf{R}^{N} \backslash \mathbf{B}}|u(x)|^{p}(|x|-1)^{-p+\beta}|x|^{1-N} d x \leq c(p) \int_{\mathbf{R}^{N} \backslash \mathbf{B}}|\nabla u(x)|^{p}(|x|-1)^{\beta}|x|^{1-N} d x,
$$

where $c(p)=\left(\frac{p}{p-1-\beta}\right)^{p}$.

Proof. Let $u \in C^{1}\left(\mathbf{R}^{N}\right)$ be a function such that $u=0$ on B. By (2.2) and Hölder's inequality, we have for $\beta / p<a<1 / p^{\prime}$

$$
\begin{aligned}
|u(x)| & \leq|x|\left(\int_{0}^{1}|\nabla u(r x)|^{p}(r|x|-1)^{a p} d r\right)^{1 / p}\left(\int_{1 /|x|}^{1}(r|x|-1)^{-a p^{\prime}} d r\right)^{1 / p^{\prime}} \\
& \leq|x|\left(\int_{1 /|x|}^{1}|\nabla u(r x)|^{p}(r|x|-1)^{a p} d r\right)^{1 / p}\left(\left[\frac{1}{1-a p^{\prime}} \frac{(r|x|-1)^{-a p^{\prime}+1}}{|x|}\right]_{1 /|x|}\right)^{1 / p^{\prime}} \\
& \leq|x|\left(\int_{0}^{1}|\nabla u(r x)|^{p}(r|x|-1)^{a p} d r\right)^{1 / p}\left(\frac{1}{1-a p^{\prime}} \frac{(|x|-1)^{-a p^{\prime}+1}}{|x|}\right)^{1 / p^{\prime}} \\
& \leq c_{1}|x|^{1 / p}\left(\int_{0}^{1}|\nabla u(r x)|^{p}(r|x|-1)^{a p} d r\right)^{1 / p}(|x|-1)^{-a+1 / p^{\prime}},
\end{aligned}
$$

where $c_{1}=\left(1-a p^{\prime}\right)^{-1 / p^{\prime}}$. Hence

$$
\begin{aligned}
& \int_{\mathbf{R}^{N} \backslash \mathbf{B}}|u(x)|^{p}(|x|-1)^{-p+\beta}|x|^{1-N} d x \\
& \leq c_{1}^{p} \int_{0}^{1}\left(\int_{\mathbf{R}^{N} \backslash \mathbf{B}}|\nabla u(r x)|^{p}(r|x|-1)^{a p}(|x|-1)^{\left(-a+1 / p^{\prime}-1\right) p+\beta}|x|^{2-N} d x\right) d r \\
& \leq c_{1}^{p} \int_{0}^{1}\left(\int_{\mathbf{R}^{N} \backslash \mathbf{B}}|\nabla u(y)|^{p}(|y|-1)^{a p}|y|^{2-N}(|y| / r-1)^{(-a p+\beta)-1} d y\right) r^{-2} d r \\
& \leq c_{1}^{p} \int_{\mathbf{R}^{N} \backslash \mathbf{B}}|\nabla u(y)|^{p}(|y|-1)^{a p}|y|^{2-N}\left(\int_{0}^{1}(|y| / r-1)^{(-a p+\beta)-1} r^{-2} d r\right) d y \\
& \leq c_{1}^{p} \int_{\mathbf{R}^{N} \backslash \mathbf{B}}|\nabla u(y)|^{p}(|y|-1)^{a p}|y|^{2-N}\left[\frac{-1}{-a p+\beta} \frac{(|y| / r-1)^{-a p+\beta}}{|y|}\right]_{0}^{1} d y \\
& \leq c_{1}^{p} \int_{\mathbf{R}^{N} \backslash \mathbf{B}}|\nabla u(y)|^{p}(|y|-1)^{a p}|y|^{2-N}\left(\frac{1}{a p-\beta} \frac{(|y|-1)^{-a p+\beta}}{|y|}\right) d y \\
& =c_{1}^{p} c_{2} \int_{\mathbf{R}^{N} \backslash \mathbf{B}}|\nabla u(y)|^{p}(|y|-1)^{\beta}|y|^{1-N} d y,
\end{aligned}
$$

where $c_{2}=(a p-\beta)^{-1}$. 
Here we see that $c_{1}^{p} c_{2}$ has the minimum $c(p)$ at $a=(1+\beta) /\left(p+p^{\prime}\right)$.

Theorem 5.5. Let $p \geq 1$. If $u \in C_{0}^{1}\left(\mathbf{R}^{N}\right)$ is a function such that $u=0$ on $\mathbf{B}$, then

$$
\begin{aligned}
& \int_{\mathbf{R}^{N} \backslash \mathbf{B}}|u(x)|^{p}|x|^{-N}(|x|-1)^{-1}|\log (|x| /(|x|-1))|^{-p} d x \\
& \leq \tilde{c}(p) \int_{\mathbf{R}^{N} \backslash \mathbf{B}}|\nabla u(x)|^{p}|x|^{p-N}(|x|-1)^{p-1} d x,
\end{aligned}
$$

where $\tilde{c}(p)=\left(\frac{p}{p-1}\right)^{p}$.

Proof. Let $u \in C_{0}^{1}\left(\mathbf{R}^{N}\right)$ be a function such that $u=0$ on B. By (2.1) and Hölder's inequality, we obtain

$$
\begin{aligned}
|u(x)| \leq & |x|\left(\int_{1}^{\infty}|\nabla u(r x)|^{p}|\log (r|x| /(r|x|-1))|^{a p}\{r(r|x|-1)\}^{p / p^{\prime}} d r\right)^{1 / p} \\
& \times\left(\int_{1}^{\infty}|\log (r|x| /(r|x|-1))|^{-a p^{\prime}}\{r(r|x|-1)\}^{-1} d r\right)^{1 / p^{\prime}} \\
\leq & |x|\left(\int_{1}^{\infty}|\nabla u(r x)|^{p}|\log (r|x| /(r|x|-1))|^{a p}\{r(r|x|-1)\}^{p / p^{\prime}} d r\right)^{1 / p} \\
& \times\left(\left[\frac{-1}{-a p^{\prime}+1}|\log (r|x| /(r|x|-1))|^{-a p^{\prime}+1}\right]_{1}^{\infty}\right)^{1 / p^{\prime}} \\
\leq & c_{1}|x||\log (|x| /(|x|-1))|^{-a+1 / p^{\prime}} \\
& \times\left(\int_{1}^{\infty}|\nabla u(r x)|^{p}|\log (r|x| /(r|x|-1))|^{a p}\{r(r|x|-1)\}^{p / p^{\prime}} d r\right)^{1 / p}
\end{aligned}
$$

when $0<a<1 / p^{\prime}$, where $c_{1}=\left(1-a p^{\prime}\right)^{-1 / p^{\prime}}$. Fubini's theorem gives

$$
\begin{aligned}
& \int_{\mathbf{R}^{N} \backslash \mathbf{B}}|u(x)|^{p}|x|^{-N}(|x|-1)^{-1}|\log (|x| /(|x|-1))|^{-p} d x \\
& \leq c_{1}^{p} \int_{1}^{\infty}\left(\int_{\mathbf{R}^{N} \backslash \mathbf{B}}|\nabla u(r x)|^{p}|x|^{-N}|\log (r|x| /(r|x|-1))|^{a p}\{r(r|x|-1)\}^{p / p^{\prime}}\right. \\
& \left.\quad \times|x|^{p}|\log (|x| /(|x|-1))|^{\left(-a+1 / p^{\prime}\right) p}(|x|-1)^{-1}|\log (|x| /(|x|-1))|^{-p} d x\right) d r \\
& =c_{1}^{p} \int_{1}^{\infty}\left(\int_{\left\{y \in \mathbf{R}^{N}:|y|>r\right\}}|\nabla u(y)|^{p}|y|^{p-N}|\log (|y| /(|y|-1))|^{a p}(|y|-1)^{p / p^{\prime}}\right. \\
& \left.\quad \times|\log (|y| /(|y|-r))|^{\left(-a+1 / p^{\prime}-1\right) p}(|y|-r)^{-1} d y\right) d r \\
& \leq c_{1}^{p} \int_{\mathbf{R}^{N} \backslash \mathbf{B}}|\nabla u(y)|^{p}|y|^{p-N}|\log (|y| /(|y|-1))|^{a p}(|y|-1)^{p / p^{\prime}} \\
& \quad \times\left(\int_{1}^{|y|}|\log (|y| /(|y|-r))|^{-a p-1}(|y|-r)^{-1} d r\right) d y
\end{aligned}
$$




$$
\begin{aligned}
\leq & c_{1}^{p} \int_{\mathbf{R}^{N} \backslash \mathbf{B}}|\nabla u(y)|^{p}|y|^{p-N}|\log (|y| /(|y|-1))|^{a p}(|y|-1)^{p / p^{\prime}} \\
& \times\left[\frac{-1}{a p}|\log (|y| /(|y|-r))|^{-a p}\right]_{1}^{|y|} d y \\
= & c_{1}^{p} c_{3} \int_{\mathbf{R}^{N} \backslash \mathbf{B}}|\nabla u(y)|^{p}|y|^{p-N}(|y|-1)^{p / p^{\prime}} d y
\end{aligned}
$$

where $c_{3}=1 /(a p)$.

It remains to see that $c_{1}^{p} c_{3}$ has the minimum $\tilde{c}(p)$ at $a=1 /\left(p+p^{\prime}\right)$.

Acknowledgment. We would like to express our deep thanks to the referees for their careful reading and useful comments.

\section{References}

[1] Adams, D. R., and L. I. HedBerg: Function spaces and potential theory. - Springer, 1996.

[2] Adams, D. R., and J. XiAO: Morrey spaces in harmonic analysis. - Ark. Mat. 50:2, 2012, $201-230$.

[3] Burenkov, V. I., A. Gogatishvili, V. S. Guliyev, and R. Ch. Mustafayev: Boundedness of the Riesz potential in local Morrey-type spaces. - Potential Anal. 35:1, 2011, 67-87.

[4] Burenkov, V. I., and V.S. Guliyev: Necessary and sufficient conditions for the boundedness of the Riesz potential in local Morrey-type spaces. - Potential Anal. 30:3, 2009, 211-249.

[5] Feichtinger, H. G., and F. Weisz: Herz spaces and summability of Fourier transforms. Math. Nachr. 281, 2008, 309-324.

[6] García-Cuerva, J., and M. J. L. Herrero: A theory of Hardy spaces associated to the Herz spaces. - Proc. London Math. Soc. 69, 1994, 605-628.

[7] Guliyev, V.S., K. Koca, and R. Ch. Mustafayev, and Ünver, T.: Some operators arising from Schwarz BVP in complementary local Morrey-type spaces on the unit disc. - J. Math. Anal. 8:1, 2017, 130-142.

[8] Guliyev, V.S., and R. Ch. Mustafaev: Fractional integrals in spaces of functions defined on spaces of homogeneous type. - Anal. Math. 24:3, 1998, 181-200 (in Russian).

[9] Hardy, G. H., J. E. Littlewood, and G. Polya: Inequalities. 2nd edition. - Cambridge, at the University Press, 1952.

[10] Herz, C.: Lipschitz spaces and Bernstein's theorem on absolutely convergent Fourier transforms. - J. Math. Mech. 18, 1968, 283-324.

[11] Kufner, A., and B. OpIC: Hardy-type inequalities. - Pitman Research Notes in Mathematics Series 219, Longman Group UK Limited, London, 1990.

[12] Kufner, A., and L.-E. Persson: Weighted inequalities of Hardy type. - World Scientific, Singapore, 2003.

[13] Maz'ya, V. G.: Sobolev spaces. - Springer, Heidelberg, 2011.

[14] Mizuta, Y.: Potential theory in Euclidean spaces. - Gakkōtosho, Tokyo, 1996.

[15] Mizuta, Y., and T. Ohno: Boundedness of the maximal operator and Sobolev's inequality on non-homogeneous central Herz-Morrey-Orlicz spaces. - Nonlinear Anal. 128, 2015, 325-347.

[16] Mizuta, Y., and T. Ohno: Sobolev's theorem and duality for Herz-Morrey spaces of variable exponent. - Ann. Acad. Sci. Fenn. Math. 39, 2014, 389-416.

[17] Mizuta, Y., and T. Ohno: Herz-Morrey spaces of variable exponent, Riesz potential operator and duality. - Complex Var. Elliptic Equ. 60:2, 2015, 211-240. 
[18] Mizuta, Y., T. Ohno, and T. Shimomura: Boundary growth of generalized Riesz potentials on the unit ball in the variable settings. - Ann. Acad. Sci. Fenn. Math. 44, 2019, 125-140.

[19] Morrey, C. B.: On the solutions of quasi-linear elliptic partial differential equations. - Trans. Amer. Math. Soc. 43, 1938, 126-166.

[20] Ragusa, M. A.: Homogeneous Herz spaces and regularity results. - Nonlinear Anal. 71, 2009, 1909-1914.

[21] Ragusa, M. A., and A. Scapellato: Mixed Morrey spaces and their applications to partial differential equations. - Nonlinear Anal. 151, 2017, 51-65.

Received 11 March $2020 \bullet$ Accepted 25 January 2021 